\title{
Tracking mobile user through adaptive beamforming for wireless cellular networks in a 2D urban environment
}

\author{
Mohamad Dosaranian-Moghadam ${ }^{1}$ and Hamidreza Bakhshi ${ }^{2}$ \\ ${ }^{1}$ Department of Electrical Engineering, Qazvin Branch, Islamic Azad University, Qazvin, Iran \\ ${ }^{2}$ Department of Electrical Engineering, University of Shahed, Tehran, Iran \\ m_dmoghadam@qiau.ac.ir'; bakhshi@shahed.ac..ir ${ }^{2}$
}

\begin{abstract}
The interference reduction capability of array antennas and the power control (PC) algorithms have been considered separately as means to increase the capacity in wireless communication networks. The capability of the smart antenna systems to track the user with the main lobe and interference with the nulls creates a significant impact on the current and future wireless sensor networks. In this paper, we use constrained least mean square (CLMS) and conjugate gradient adaptive beam-forming (CGBF) algorithms for narrowband adaptive beam-forming for tracking mobile user in a 2D urban environment without using PC algorithm. The CLMS algorithm is capable of efficiently adapting according to the environment and able to permanently maintain the chosen frequency response in the look direction while minimizing the output power of the array. In addition, with the CGBF algorithm, the desired users' signal in an arbitrary path is passed and the inter-path interference (IPI) is canceled in other paths in each RAKE finger. The adaptability of the algorithms is closely observed for uniformly spaced linear array. Also in this paper, we present switched-beam (SB) technique. In the SB technique by using a number of fixed, independent, or directional antennas we increase the uplink capacity of the wireless systems. Simulation results indicate that the SB technique is able to considerably increase the signal to interference plus noise ratio (SINR) of mobile user in comparison with other algorithms. In addition, we observe that the SINR in the CLMS algorithm is lower than the CGBF algorithm. Finally, we discuss perfect power control and path loss parameter in urban environments and their effects on system capacity by simulations.
\end{abstract}

Keywords: Antenna, Switched-beam, Power control, Urban signal propagation simulator, Wireless cellular network.

\section{Introduction}

The use of smart antennas is expected to have a significant impact on future wireless communications to meet the projected perspective of future communication networks. A major reason to use smart antenna in wireless communication is its capability to intelligently respond to the unknown interference environment in real time. The process of formation of nulls in the direction of interference and strong beams in the direction of desired user is called adaptive array processing. These systems are called adaptive beam-forming system and consist of spatially disposed sensor elements connected to a single channel or to a multi channel adaptive processor. The term adaptive beam-forming is also referred as smart antennas. Adaptive antenna array can be used to eliminate the directional interference by adaptive canceling and therefore to improve the signal to interference plus noise ratio (SINR). Steering capability of adaptive array depends on processing algorithms for null steering. Such algorithms are called adaptive algorithm. In wireless communications, smart antennas are used due to their ability to separate the desired signal from interfering signals. By knowing the direction of the desired signals, they are able to adjust the antenna pattern intelligently by adjusting the weights of the adaptive algorithm (Riba et al. 1997; Liberti \& Rappaport, 1999; Shakir \& Durrani, 2007).

Propagation model in 2D urban environment

Because of using 2D urban structure in this paper, for computing yield for the path between a mobile set (MS) and base station (reverse link), propagation model is dramatized in urban environments. Accordingly, with signal propagation simulator (USPS) software, mobile set antenna radiate beams which diffuse in all directions and parts of the beams reach to base station (BS). In this software, delivered beam from mobile set by the time of collision to an obstacle like a wall surface or a building, reflects to a new angle and continues its path, which is called reflection phenomena. In condition that radiated beam is conflicted to an obstacle edge, then diffraction phenomena is happened and diffracting point is diffusing

Fig. 1. Diffraction phenomena and reflection phenomena in the USPS software (LoS and Non-LoS paths) for a 2D urban environment in reverse link.

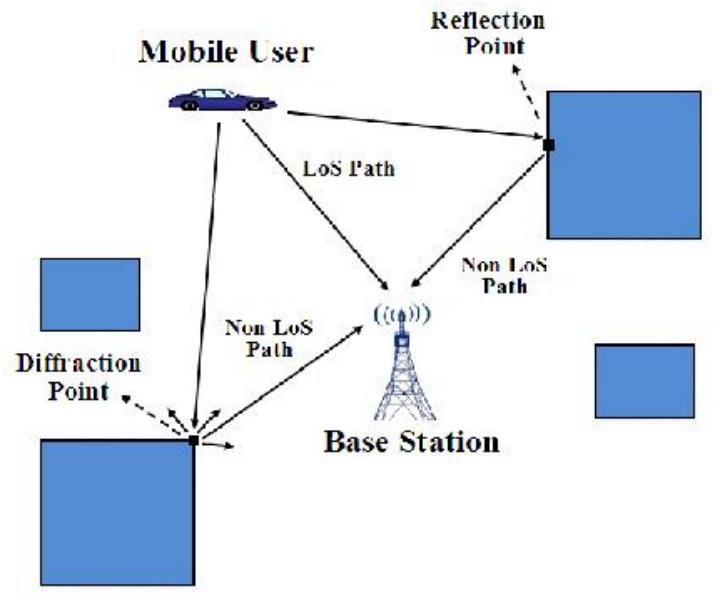


new beams to all directions like a transmitter. All reflected beams will stay in the environment as long as their power is not reduced to a threshold limit. Fig.1 shows both phenomena for LoS and non-LoS paths in reverse link. On the other hand, in this software, the channel is modeled with lognormal distributed shadowing. Accordingly, every pixel of the environment stores all environmental information like receiving power and angle for each path (Rashid-Farrokhi et al., 1998; Dosaranian Moghadam et al., 2009; Dosaranian -Moghadam et al., 2009; Dosaranian-Moghadam et al., 2010).

According to the above dramatization, we could see that because of LoS in un-urban environment, only one signal is delivered from each user to a receiver, while in function and because of the elimination phenomena in urban environments, beside to signals which are delivered to line sight, signals which have difference in phase or domain with this signal are also received by the receiver.

\section{System model}

Fig. 2. Antenna array and beamformer (Peterson et al., 1995).

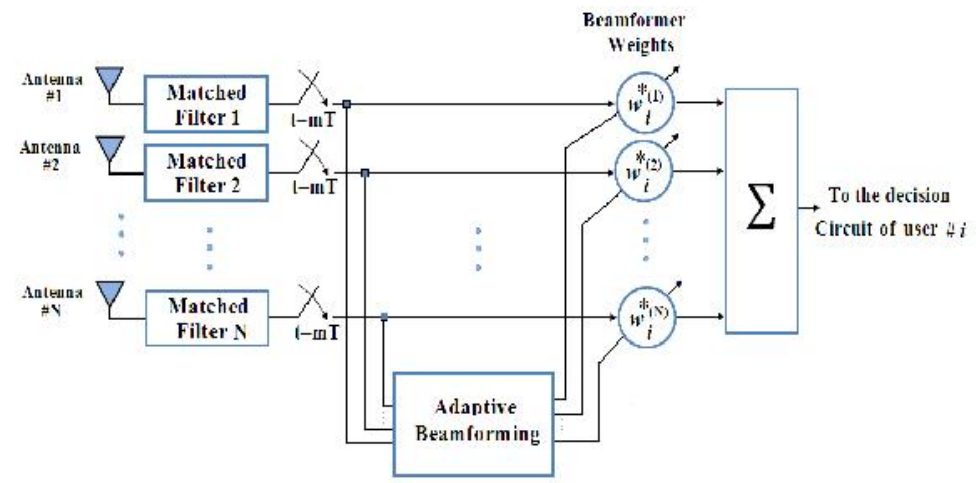

An adaptive antenna array consists of a set of antennas, designed to receive signals radiating from some specific directions and attenuate signals radiating from other directions of no interest. The outputs of array elements are weighted and added by a beam-former, as shown in Fig.2, to produce a directed main beam and adjustable nulls. In order to reject the interference, the beam-former has to place its nulls in the directions of sources of interference, and steer to the direction of the target signal by maintaining constant gain at this direction (Rashid-Farrokhi et al., 1998). In this paper, we focus on the uplink communication path in a single-cell wireless system with $M$ users and only one BS. In addition, we use a uniform linear array (ULA) of $N$ antenna elements in the base station.

Accordingly, the received signal vector in BS can be written as (Rashid-Farrokhi et al., 1998; DosaranianMoghadam et al., 2010).

$$
\mathbf{x}(t)=\sum_{i=1}^{M} \sqrt{p_{i}^{\prime}} s_{i}\left(t-\tau_{i}\right) \mathbf{v}_{i}(\theta)+\mathbf{n}(t)
$$

where $s_{i}(t)$ is the message signal transmitted from the th user (desired/mobile user); $p_{i}^{\prime}$ is the received power of the $i^{\text {th }}$ user in BS which is equal to $p_{i} G_{i}$ where $p_{i}$ and $G_{i}$ denote the transmitted power of the th $i^{\text {th }}$ user, and the link gain between user $i$ and BS in LoS path (or the best nonLoS path if LoS path is not exist), as shown in Fig.1, respectively. It should be mentioned that for perfect power control (PPC), the received power is $p_{i}^{\prime}=P^{\prime}=E_{b} / T$ for all users connected to similar base station, where $T$ and $E_{b}$ are bit period and the energy per bit for all users, respectively, while in this paper for the case of without power control (PC) algorithm, we assume the transmitted power is $p_{i}=P$ for all users. Also, $\mathbf{n}(t)$ is the thermal noise vector at the input of antenna array at BS and $\tau_{i}$ is the corresponding time delay. For simplicity, we further assume that the time delays for all uses are equal to 0 . Also the $N \times 1$ vector $\mathbf{v}_{i}(\theta)$ is the array response for user $i$ and can be written as follows (Rashid-Farrokhi et al., 1998).

$$
\mathbf{v}_{i}(\theta)=\sum_{l=1}^{L_{i}} \sqrt{\alpha_{i, l}} \mathbf{a}_{i}\left(\theta_{i, l}\right)
$$

where $L_{i}$ is the number of paths between user $i$ and BS in USPS; $\theta_{i, l}$ is the direction of arrival (DoA) in the $f^{\text {th }}$ path for user $i$; $\alpha_{i, l}$ is the normalized attenuation by the best link gain $\left(G_{i}\right)$ in USPS between user $i$ and BS in the th path, therefore $0<\alpha_{i, l} \leq 1$. Also, response of the base station array to the direction $\theta_{i, l}$ is given by

$$
\mathbf{a}_{i}\left(\theta_{i, l}\right)=\left[1 \exp \left(-j \tau_{\theta_{i, l}}\right) \ldots \exp \left(-j(N-1) \tau_{\theta_{i, l}}\right)\right]^{T}
$$

where $j=\sqrt{-1}$ and $\tau_{\theta_{i, l}}=(2 \pi d / \lambda) \sin \theta_{i, l}$, in which $\lambda$ is signal wavelength and $d$ is the distance between the antenna elements that for avoid the spatial aliasing should be defined as $d=0.5 \lambda$.

In non-spread spectrum systems, the transmitted signal is given by (Rashid-Farrokhi et al., 1998).

$$
s_{i}(t)=\sum_{m} b_{i}(m) g(t-m T)
$$

where $b_{i}(m)$ is the th user information bit stream with a bit period of $T$ and $g(t)$ is the pulse-shaping filter impulse response. It has been shown that the output of a matched filter sampled at the symbol intervals is a sufficient statistic for the estimation of the transmitted signal. The matched filter is sampled at $t=m T$

$\mathbf{x}(m)=\left.\mathbf{x}(t) * g^{*}(-t)\right|_{t=m T}$

Hence, the received signal at the output of the matched filter is given by
"Wireless cellular network" http://www.indjst.org
M.D-Moghadam \& H.Bhakshi Indian J.Sci.Technol. 


$$
\begin{aligned}
\mathbf{x}(m) & =\sqrt{p_{i} G_{i}} b_{i}(m) \mathbf{v}_{i}(\theta)+\sum_{\substack{k=1 \\
k \neq i}}^{M} \sqrt{p_{k} G_{k}} b_{k}(m) \mathbf{v}_{k}(\theta)+\mathbf{n}(m) \\
& =\sqrt{p_{i} G_{i}} b_{i}(m) \mathbf{v}_{i}(\theta)+\mathbf{I}_{i}(m)+\mathbf{n}(m)
\end{aligned}
$$

where $\mathbf{I}_{i}(m)$ is the interference for user $i$ in BS and $\mathbf{n}(m)=\left.\mathbf{n}(t) * g^{*}(-t)\right|_{t=m T}$ ( Dosaranian-Moghadam et al., 2010).

The types of adaptive beamforming algorithms

Constrained LMS algorithm: Constrained LMS algorithm is a gradient based algorithm to minimize the total processor output power, based on the look direction constraint. The adaptive algorithm is designed to adapt efficiently in agreement with the environment and able to permanently preserve the desired frequency response in the look direction while minimizing the output power of the array. The combined form of the constraint is called constraint for narrowband beam-forming (Rashid-Farrokhi et al., 1998; Shakir \& Durrani, 2007; Xinyu et al., 2008; Gotsis et al., 2009).

This form consider a narrowband beam-former where the narrowband signal from each element of smart antenna are multiplied by the complex weight calculated by using narrowband adaptive beam-forming algorithm, and then summed to produce the output of the array. The definition of the complex weights of this beam-former in the $m^{\text {th }}$ iteration for the th ${ }^{\text {th }}$ user is as follows (Shakir \& Durrani, 2007; Xinyu et al., 2008):

$\mathbf{w}_{i}(m)=\left[w_{i}^{(1)}(m) w_{i}^{(2)}(m) \ldots w_{i}^{(N)}(m)\right]^{T}$

Accordingly, the output of the array in the $m^{\text {th }}$ iteration for user $i$ is given by

$$
y_{i}(m)=\mathbf{w}_{i}^{H}(m) \mathbf{x}(m)
$$

The expected output power of the array in the $m^{\text {th }}$ iteration is given by

$$
\begin{aligned}
\mathrm{E}\left(\left|y_{i}(m)\right|^{2}\right)= & \mathrm{E}\left(y_{i}(m) y_{i}^{*}(m)\right) \\
& =\mathrm{E}\left(\mathbf{w}_{i}^{H}(m) \mathbf{x}(m) \mathbf{x}^{H}(m) \mathbf{w}_{i}(m)\right)=\mathbf{w}_{i}^{H}(m) \mathbf{R}_{x x} \mathbf{w}_{i}(m)
\end{aligned}
$$

where $\mathrm{E}($.$) is denoted the expectation and \mathbf{R}_{x x}$ is the correlation matrix of the received signal vector $\mathbf{x}(m)$.

A real-time constrained least mean square (CLMS) algorithm for determining the optimal weight vector for user $i$ is (Shakir \& Durrani, 2007; Xinyu et al., 2008).

$$
\left\{\begin{array}{l}
\mathbf{w}_{i}(m+1)=\mathbf{w}_{i}(m)+\mu g\left(\mathbf{w}_{i}(m)\right) \\
\mathbf{w}_{i}^{H} \mathbf{a}_{i}\left(\theta_{i, \mathrm{~g}_{\max }}\right)=1
\end{array}\right.
$$

where $\mathbf{a}_{i}\left(\theta_{i, \mathrm{~g}_{\max }}\right)$ denotes spatial response of the array for user $i$ in the best path gain, i.e., $\alpha_{i, l}=1$, and $\mathbf{w}_{i}(m+1)$ is the new weight computed at the $(m+1)$ th iteration for user $i$. Also, the variable scalar $\mu$ denotes a positive scalar (gradient step size) that controls the convergence characteristic of the algorithm, that is, how fast and how close the estimated weights approach the optimal weights, and $g\left(\mathbf{w}_{i}(m)\right)$ denotes an unbiased estimate of the gradient of the power surface $\left(\mathbf{w}_{i}(m)^{H} \mathbf{R}_{x x} \mathbf{w}_{i}(m)\right.$ which is the expected output power of the array) with respect to $\mathbf{w}_{i}(m)$ after the $m^{\text {th }}$ iteration. The algorithm is "constrained" because the weight vector satisfies the constraint at each iteration, that is $\mathbf{w}_{i}^{H} \mathbf{a}_{i}\left(\theta_{i, g_{\max }}\right)=1$. Rewrite the CLMS algorithm as follows (Litva \& KwokYeung, 1996; Haykin, 1996; Xinyu et al., 2008),

$$
\mathbf{w}_{i}(m+1)=\boldsymbol{\beta}_{i}\left(\mathbf{w}_{i}(m)+\mu g\left(\mathbf{w}_{i}(m)\right)\right)+\frac{\mathbf{a}_{i}\left(\theta_{i, g_{\max }}\right)}{N}
$$

where

$\boldsymbol{\beta}_{i}=\mathbf{I}-\frac{\mathbf{a}_{i}\left(\theta_{i, g_{\max }}\right) \mathbf{a}_{i}\left(\theta_{i, g_{\max }}\right)^{H}}{N}$

The gradient of $\mathbf{w}_{i}^{H}(m) \mathbf{R}_{x x} \mathbf{w}_{i}(m)$ with respect to $\mathbf{w}_{i}(m)$ is given by

$$
g\left(\mathbf{w}_{i}(m)\right) \stackrel{\Delta}{=}-\frac{\partial}{\partial \mathbf{w}_{i}^{*}}\left(\mathbf{w}_{i}^{H}(m) \mathbf{R}_{x x} \mathbf{w}_{i}(m)\right)=-2 \mathbf{R}_{x x} \mathbf{w}_{i}(m)
$$

and its computation using this expression requires knowledge of $\mathbf{R}_{x x}$, which normally is not available in practice. For a standard LMS algorithm, an estimate of the gradient at each iteration is made by replacing $\mathbf{R}_{x x}$ by its noise sample $\mathbf{x}_{i}(m+1) \mathbf{x}_{i}(m+1)^{H}$ available at time instant $(m+1)$, leading to

$$
g\left(\mathbf{w}_{i}(m)\right)=-2 \mathbf{x}_{i}(m+1) y_{i}^{*}(m)
$$

The CLMS is a fast convergence algorithm. However, it is drastically sensitive to the mismatch in the direction of arrival. Meanwhile, the weights estimated by the standard algorithm are sensitive to the signal power, requiring a lower step size in the presence of a strong signal for the algorithm to converge, which in turn regarding the decrease of mis-adjustment error, the convergence time is increased (Xinyu et al., 2008).

Consider the problem of beam-forming as to maximize the SINR for a specific link, which is equivalent to minimizing the interference at the receiver of that link. In order to minimize the variance or average power at the output of the beam-former subject to maintaining unity gain at the direction of the mobile user signal. Accordingly, we can rewrite the average output power for user $i$ as (Rashid-Farrokhi et al., 1998),

$\zeta_{i}=\hat{\mathbf{w}}_{i}^{H} \mathbf{R}_{x x} \hat{\mathbf{w}}_{i}$

where $\hat{\mathbf{w}}_{i}$ is the optimum weight vector for user $i$ with CLMS algorithm. If the message signals $s_{i}(t)$ are uncorrelated and zero mean, the correlation matrix $\mathbf{R}_{x x}$ is given by

$$
\begin{aligned}
\mathbf{R}_{x x}= & p_{i} G_{i} \mathbf{v}_{i}(\theta) \mathbf{v}_{i}^{H}(\theta)+\sum_{\substack{k=1 \\
k \neq i}}^{M} p_{k} G_{k} \mathbf{v}_{k}(\theta) \mathbf{v}_{k}^{H}(\theta)+\sigma_{n}^{2} \mathbf{I} \\
& =p_{i} G_{i} \mathbf{v}_{i}(\theta) \mathbf{v}_{i}^{H}(\theta)+\boldsymbol{\Phi}_{i}
\end{aligned}
$$

Research article

COIndian Society for Education and Environment (iSee)
"Wireless cellular network" http://www.indjst.org
M.D-Moghadam \& H.Bhakshi Indian J.Sci.Technol. 
where

$$
\boldsymbol{\Phi}_{i}=\sum_{\substack{k=1 \\ k \neq i}}^{M} p_{k} G_{k} \mathbf{v}_{k}(\theta) \mathbf{v}_{k}^{H}(\theta)+\sigma_{n}^{2} \mathbf{I}
$$

is the correlation matrix of unwanted signals for user $i$ in $\mathrm{BS}$, and $\sigma_{n}^{2}$ is the noise power at the input of each array element.

Combining (15)-(17), we obtain the received signal plus interference power as a function of weight vector

$\zeta_{i}=p_{i} G_{i}\left|\hat{\mathbf{w}}_{i}^{H} \mathbf{v}_{i}(\theta)\right|^{2}+\sum_{\substack{k=1 \\ k \neq i}}^{M} p_{k} G_{k} \hat{\mathbf{w}}_{i}^{H} \mathbf{v}_{k}(\theta) \mathbf{v}_{k}^{H}(\theta) \hat{\mathbf{w}}_{i}+\sigma_{n}^{2} \hat{\mathbf{w}}_{i}^{H} \hat{\mathbf{w}}_{i}$

Accordingly, the SINR at BS for user $i$ can be written as follows (Rashid-Farrokhi et al., 1998).

$$
\operatorname{SINR}_{i}(\hat{\mathbf{w}}, \mathbf{v}(\theta))=\frac{p_{i} G_{i}\left|\hat{\mathbf{w}}_{i}^{H} \mathbf{v}_{i}(\theta)\right|^{2}}{\sum_{\substack{k=1 \\ k \neq i}}^{M} p_{k} G_{k}\left|\hat{\mathbf{w}}_{i}^{H} \mathbf{v}_{k}(\theta)\right|^{2}+\sigma_{n}^{2} \hat{\mathbf{w}}_{i}^{H} \hat{\mathbf{w}}_{i}}
$$

It should be mentioned that for the array antenna weight vector elements in the CLMS algorithm and for big $\mu$, will converge after a few iteration (is approximately equal to the number of beamformer weights, i.e., $m=N$ ), (Dosaranian et al., 2009; Haykin,1996; DosaranianMoghadam et al., 2010; Dosaranian-Moghadam et al., 2010; Dosaranian-Moghadam et al., 2010; DosaranianMoghadam et al., 2011). Moreover it is obvious that, without power control algorithm, the calculated optimal weight vector does not guarantee desirable SINR for the mobile user. In simulation section, we will consider perfect power control for increasing SINR of the mobile user.

Conjugate gradient adaptive beam forming: It is well known that an array of $N$ weights has $N-1$ degree of freedom for adaptive beam-forming. This means that with an array of $N$ weights, one can generate $N-1$ pattern nulls and a beam maximum in desired directions. Accordingly, we assume a single-cell wireless system, with $M$ users connected to the base station. Thus, the number of interference signals is $\mathrm{K}=L M-1$ that $L$ is the number of paths for each link. To nullify all of these interference signals, one would have to have $\mathrm{K}+1$ weights, which is not practical. So, we focus only on the $L$ paths of the desired user (inter-path interference). Thus, the minimum number of the antenna array weights is $L$ where, typically, $L$ varies from 2 to 6 (RashidFarrokhi et al., 1998).

In this paper, we use the gradient adaptive beamforming (CGBF) algorithm that is used based on orthogonal principle (Mohamed \& Dunham 1999; Mohamed \& Dunham, 2002; Dosaranian-Moghadam et al., 2010; Dosaranian-Moghadam et al., 2010).
Vol. 5 No. 4 (Apr 2012)

ISSN: 0974- 6846

On this basis, a set of vectors $\mathbf{w}_{k}$ is to be selected such that they are $\mathbf{A}$-orthogonal, i.e., $\left\langle\mathbf{A w}_{k}, \mathbf{A w}_{k^{\prime}}\right\rangle=0$ for $k \neq k^{\prime}$. In this algorithm, at each base station, an antenna array of $S$ sensors and $N$ weights is employed, where $S=2 N-1$ for $n=-(N-1), \ldots,-1,0,1, \ldots,+(N-1)$, to receive signals from all users (see DosaranianMoghadam et al., 2010 Dosaranian-Moghadam et al.,2010 and for more details). Note that in CGBF adaptation algorithm, unlike other adaptation algorithms, for example, the CLMS algorithm, the number of weights is less than the number of sensors. Accordingly, the optimum weights of th $f^{\text {th }}$ branch of the RAKE receiver for the CGBF algorithm at time $m$ are obtained by minimizing (Mohamed \& Dunham, 1999; Mohamed \& Dunham, 2002; Dosaranian-Moghadam et al., 2010; Dosaranian-Moghadam et al., 2010)

$\left\|\boldsymbol{\Psi}_{i}^{(j)}(m)\right\|^{2}=\boldsymbol{\Psi}_{i}^{H(j)}(m) \boldsymbol{\Psi}_{i}^{(j)}(m)$

where

$\boldsymbol{\Psi}_{i}^{(j)}(m)=\mathbf{A}_{i} \mathbf{w}_{i}^{(j)}(m)-\mathbf{y}_{i}^{(j)}$

and using (6),

$\mathbf{A}_{i}=\left[\begin{array}{ccc}x_{-}(N-1) & \ldots & x_{0} \\ \cdot & \cdot & \cdot \\ x_{0} & \ldots & x_{+(N-1)}\end{array}\right]$

is the $N \times N$ signal matrix. Also,

$\mathbf{y}_{i}^{(j)}=\left[\begin{array}{lllll}e^{-j(N-1) \theta / 2} & \ldots & 1 & \ldots & e^{+j(N-1) \theta / 2}\end{array}\right]^{T}$

and

$\mathbf{w}_{i}^{(j)}(m)=\left[w_{i, 0}^{(j)}(m) w_{i, 1}^{(j)}(m) \quad \ldots \quad w_{i, N-1}^{(j)}(m)\right]^{T}$

are the excitation and weight vectors $(N \times 1)$ for desired user in the th ${ }^{\text {th }}$ path, respectively.

It should be mentioned that the CGBF algorithm has two main characteristics (Mohamed \& Dunham, 2002):

1.This algorithm can produce a solution of the matrix equation very efficiently and converge in a finite number of iterations (the number of beam-former weights).

2. Convergence is guaranteed for any possible condition of the signal matrix, according to (DosaranianMoghadam et al., 2010).

According to the algorithm of CGBF, the updated value of the weight vector for desired user in the $f^{\text {th }}$ path at time $m+1$ is computed by using the simple recursive relation (Mohamed \& Dunham 1999; Mohamed \& Dunham, 2002),

$$
\mathbf{w}_{i}^{(j)}(m+1)=\mathbf{w}_{i}^{(j)}(m)+\kappa_{i}^{(j)}(m) \boldsymbol{\beta}_{i}^{(j)}(m)
$$

where

$$
\begin{gathered}
\boldsymbol{\kappa}_{i}^{(j)}(m)=\left\|\mathbf{A}_{i}^{H} \boldsymbol{\Psi}_{i}^{(j)}(m)\right\|^{2} /\left\|\mathbf{A}_{i} \boldsymbol{\beta}_{i}^{(j)}(m)\right\|^{2} \\
\boldsymbol{\Psi}_{i}^{(j)}(m+1)=\boldsymbol{\Psi}_{i}^{(j)}(m)+\kappa_{i}^{(j)}(m) \boldsymbol{\beta}_{i}^{(j)}(m) \\
\boldsymbol{\beta}_{i}^{(j)}(0)=-\mathbf{A}_{i}^{H} \boldsymbol{\Psi}_{i}^{(j)}(0) \quad(26 \mathrm{c})
\end{gathered}
$$

"Wireless cellular network" http://www.indjst.org 
Accordingly, we can rewrite (18) for the CGBF algorithm as follows.

$$
\begin{aligned}
& \zeta_{i}^{j}=p_{i} G_{i}\left|\hat{\mathbf{w}}_{i}^{H(j)} \mathbf{v}_{i}^{(j)}(\theta)\right|^{2}+ \\
& \sum_{\substack{k=1 \\
k \neq i}}^{M} p_{k} G_{k} g_{i}^{(j)}(\theta) \hat{\mathbf{w}}_{i}^{H} \mathbf{v}_{k}(\theta) \mathbf{v}_{k}^{H}(\theta) \hat{\mathbf{w}}_{i}+\sigma_{n}^{2} \hat{\mathbf{w}}_{i}^{H} \hat{\mathbf{w}}_{i}
\end{aligned}
$$

where

$$
\begin{aligned}
& \mathbf{v}_{i}^{(j)}(\theta)=\sum_{l=1}^{L_{i}} \sqrt{\alpha_{i, l}} \mathbf{a}_{i}\left(\theta_{i, l}\right) g_{i}^{(j)}(\theta) \\
& =\underbrace{\sqrt{\alpha_{i, 0}} \mathbf{a}_{i}\left(\theta_{i, 0}\right)+\ldots+\sqrt{\alpha_{i, j-1}} \mathbf{a}_{i}\left(\theta_{i, j-1}\right)}_{\text {IPI }=\mathbf{0}} \\
& +\sqrt{\alpha_{i, j}} \mathbf{a}_{i}\left(\theta_{i, j}\right)+\underbrace{\sqrt{\alpha_{i, j+1}} \mathbf{a}_{i}\left(\theta_{i, j+1}\right) \ldots+}_{\text {IPI }=\mathbf{0}}
\end{aligned}
$$

$$
=\sqrt{\alpha_{i, j}} \mathbf{a}_{i}\left(\theta_{i, j}\right)
$$

and

$$
g_{i}^{(j)}(\theta)=\left[\begin{array}{llll}
e^{-j(N-1) \theta / 2} & \ldots 1 \ldots e^{+j(N-1) \theta / 2}
\end{array} \times \mathbf{w}_{i}^{(j)}\right.
$$

is the magnitude response of the $\mathrm{j}^{\text {th }}$ beam-former for user $i$ toward the direction of arrival $\theta$ and $\mathbf{w}_{i}^{(j)}$ is the $f^{\text {th }}$ beam-former's weight vector for user $i$.

Accordingly, the desired user resolvable directions are fed to the conjugate gradient beam-former to cancel out the inter-path interference (IPI) from other directions.

Fig.3. 36 beams in each base station with switched-beam technique

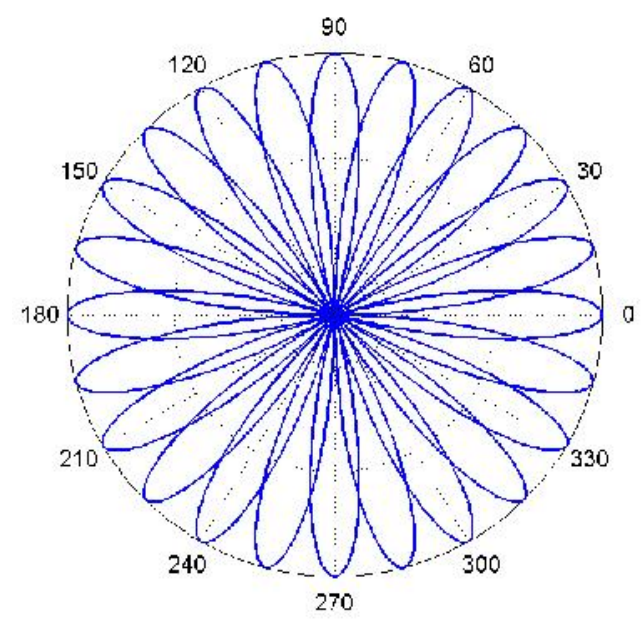

Research article

CIndian Society for Education and Environment (iSee)
Also, the output signals from the beam-former with conventional maximal ratio combining (MRC) are combined and then are fed into the decision circuit of the

Fig.4. Select of beam for two users in two paths with switched-beam technique

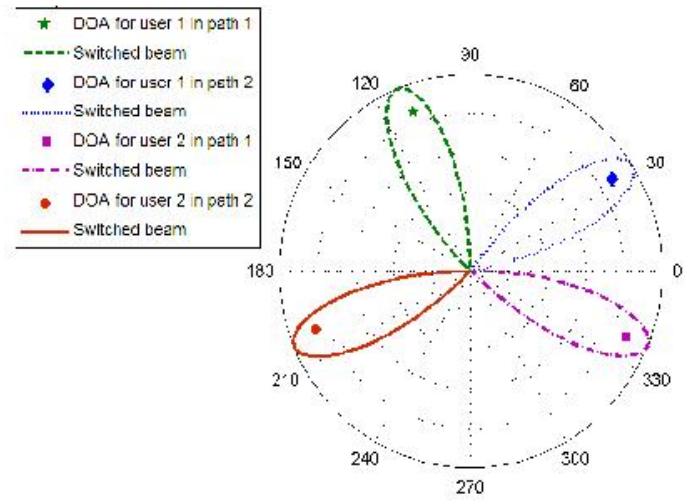

mobile user. Hence, by the CGBE algorithm, mobile users' signal in an arbitrary path is passed and the IPI is canceled in other paths in each RAKE finger, while the IPI is not removed in the CLMS algorithm (Peterson et al., 1995).

The switched-beam technique: One simple alternative to the fully adaptive antenna is the switched-beam architecture in which the best beam is chosen from a number of fixed steered beams. Switched-beam systems are technologically the simplest and can be implemented by using a number of fixed, independent, or directional antennas (Gotsis et al., 2009). We list the conditions of the switched-beam (SB) technique for this paper as follows (Dosaranian-Moghadam et al., 2010).

1. According to Fig.3, beams coverage angle is $30^{\circ}$ and overlap between consecutive beams is $20^{\circ}$. Thus each base station has 36 beams.

Fig.5. Two-dimensional map of the University of Toronto campus area and placing interference users (M-1), mobile user

( $a, b, c$, and $d$ positions) and base- station contain array antenna in map center (Dosaranian-Moghadam et al., 2009).

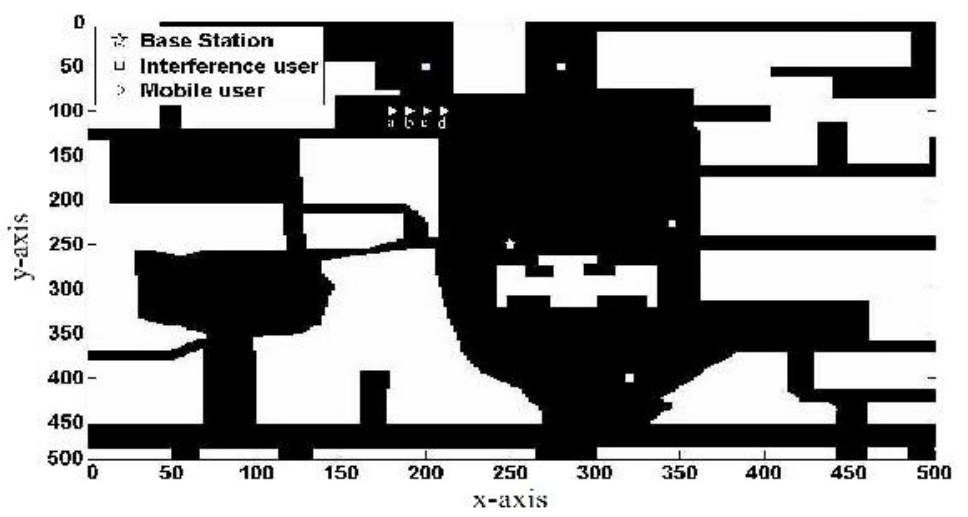

"Wireless cellular network" http://www.indjst.org
M.D-Moghadam \& H.Bhakshi Indian J.Sci.Technol. 
2. According to Fig.4, each user can use one beam for each of its path to communicate with a base station at any time.

Equal Sectoring Method: One simple method used to sectorize a cell is equal sectoring; in which all sectors have the same coverage angle. In this paper, we assume 12 sectors for each base station with sector angle $30^{\circ}$ for the equal sectoring (ES) method (Dosaranian-Moghadam et al., 2010).

Fig.6. Normalized polar beam pattern plot for uniformly spaced linear array with uniform inter-element spacingd $=\lambda / 2, N=10$ elements in antenna array in four positions ( $a, b, c$ and $d$ positions as Fig. 4)
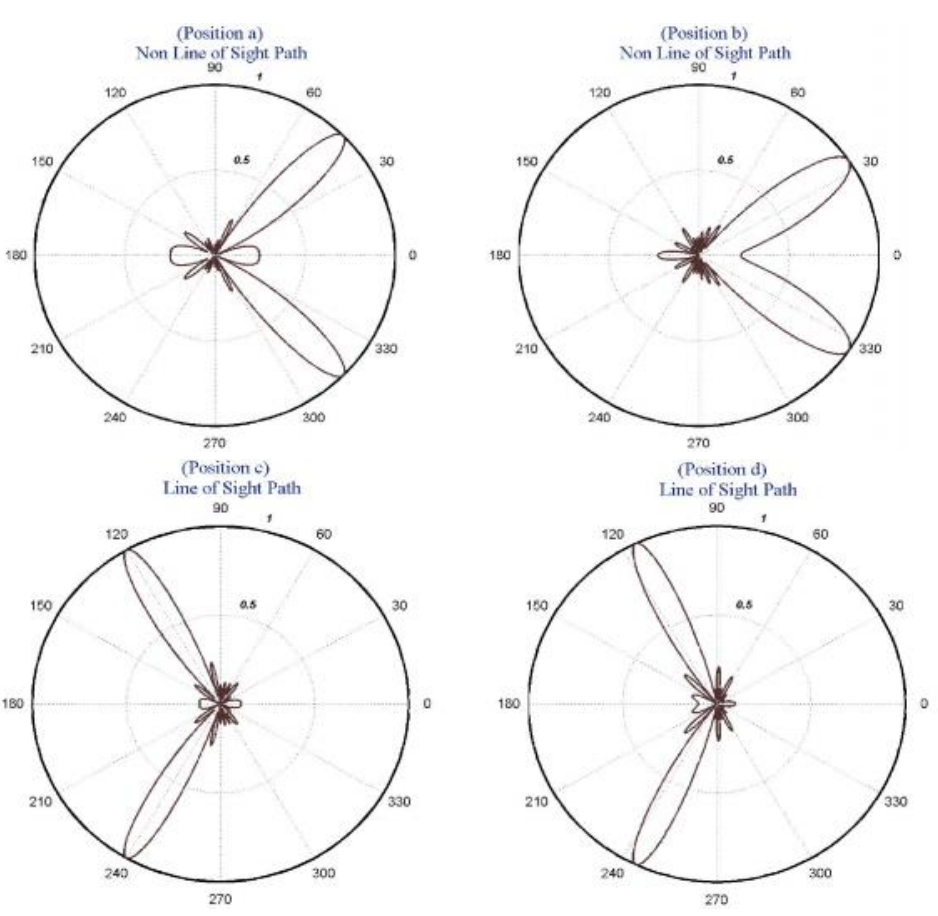

Fig.7. Normalized polar beam pattern plot with $N=10$ elements in antenna array and for uniformly spaced linear array with uniform inter-element spacing (a) $d=0.75 \lambda$ (b) $d=0.2 \lambda$ ( $d$ position as Fig. 5)

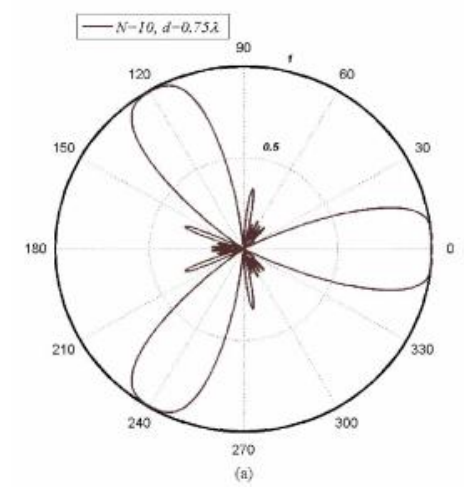

Research article COIndian Society for Education and Environment (iSee)

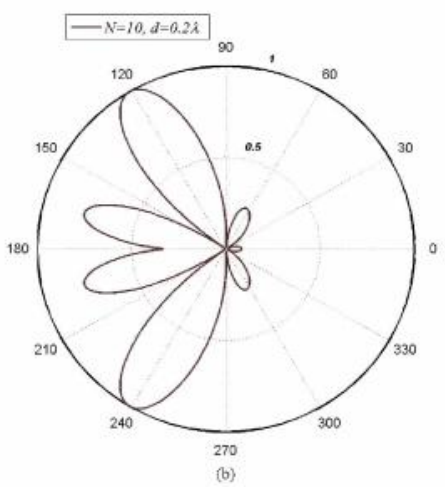

"Wireless cellular network" http://www.indjst.org
Vol. 5 No. 4 (Apr 2012)

ISSN: 0974- 6846

\section{Simulation results}

In this section, we will track a mobile user by adaptive beamforming algorithm. For this purpose, we chose part of Two-dimensional map of the University of Toronto campus area in Canada as shown in Fig.5 (Dosaranian Moghadam et al., 2009; Corazza et al., 1998). According to this figure, we observe beside main mobile user, four interference users $(M-\eta)$ in a stable positioning situation are also sending information to base station equipped to an array antenna in reverse link. Base station disposition place, interference users and also mobile user changing place are also showed in four positions as Fig.5. For this and also according to the description in Section 2, sending information by all users to base station is performed by dramatizer USPS. Also, we assume $L_{i}=L=4$ resolvable propagation paths for all users for all links; resolution, path loss parameter, and variance of the log-normal shadow fading in USPS $R=1, L_{p}=0.005 \mathrm{~dB} / \mathrm{m}$ and $4 \mathrm{~dB}$ respectively.

Also, we assume that the variance of the noise $\left(\sigma_{n}^{2}\right)$ for every element is assumed to be equal to 0.01 in all our simulations.

In Fig. 6 for $\mu=0.005, d=\lambda / 2$ and $N=10$, tracking mobile user for the CLMS algorithm in four positions $(a, b$ and $c, d$ are non-LoS and LoS positions, respectively) are shown as Fig.5. According to this figure, by changing mobile user place, vectors for array components weights are arranged comparatively that considering produced beam pattern, difference between reference signal and received signal in output array antenna components is minimized. Regarding Fig.6, there is a line of sight direction in the situations of $c$ and $d$, while, the situations of $a$ and $b$ have a nonline of sight direction.

Fig.7 shows the capability of beamforming and nulling of antenna array with the different inter-element spacing $(d=0.75 \lambda$ and $d=0.2 \lambda)$ in $d$-position as Fig.5 for $\mu=0.005$ and $N=10$. Similar to what we see in Fig.6, we observe that inter-element spacing is very important criteria for array formation. In order to avoid the spatial aliasing, the optimum range for the array design should be defined as $d=0.5 \lambda$. In addition, Fig. 8 presents the capability of beam-forming for $d=0.75 \lambda$ and for different values of the number of the components of the array antenna $(N)$. This figure shows that the beam width for the case of $N=20$ is narrower than that for the case of $N=10$, thus, the SINR of mobile user for $N=20$ is higher than that for $N=10$.

In Table 1, for the CLMS algorithm and $\mu=0.005$, the relation between the distance of the components $(d)$ and the number of the components of the array antenna $(N)$ for the beam width in the main lobe look direction and for the maximum exposure rate (MER) in 
Fig.8. Normalized polar beam pattern plot with uniform interelement spacingd $=0.75 \lambda$ and for the number of elements in antenna array (a) $N=10$ (b) $N=20$

(d-position as Fig. 5)
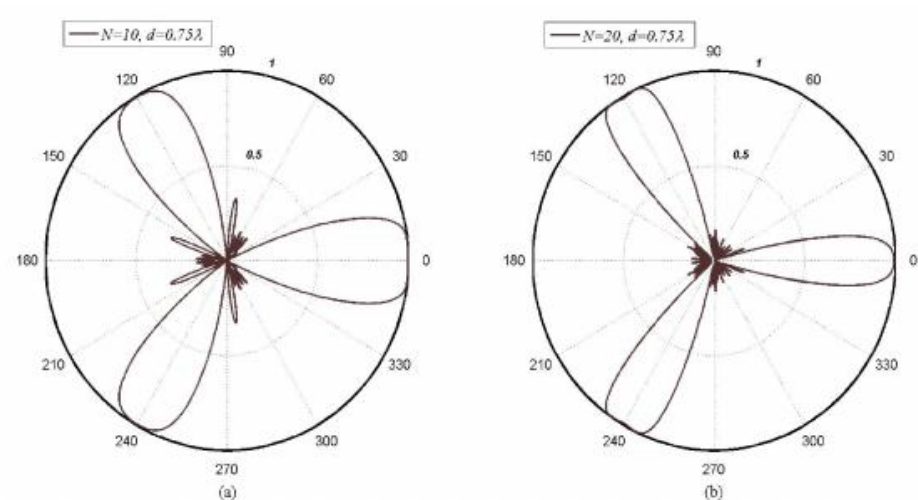

the side lobe direction has been depicted. Based on the results, the bigger the number of the components, the narrower the beam width to the main user will be, and this leads to the decrease of the interference signal and the increase of SINR for the intended user. Moreover, it is obvious that the beam width for two states, $d=0.4 \lambda, 0.5 \lambda$,

is narrower than the other states, and also the phenomenon of spatial aliasing has not been occurred (Dosaranian -Moghadam et al., 2009) for more details.

In Fig.9, the SINR of mobile user in reverse link without power control algorithm versus the number of interference users $(M-1)$ is compared. In this simulation, we use the SB, CGBF, CLMS, and ES methods for $N=15,20, d=0.5 \lambda, 0.75 \lambda$, path loss parameter $L_{P}=0.005 \mathrm{~dB} / \mathrm{m}$, and $\mu=0.005$. Also in this simulation, we consider the effect of perfect power control on the SINR of mobile user. This figure shows that the SINR for mobile user with SB technique is higher than that with the other methods. On the other hand, the figure shows the SINR for mobile user with CGBF algorithm is higher than that with CLMS algorithm, because in CGBF algorithm the IPI is removed whereas in CLMS algorithm it is not canceled. Also, we observe that the SINR in the ES method is lower than that in the other methods, because

Table 1. Performance of smart antennas with variation in number of elements $(N)$ and inter-element spacing $(d)$

\begin{tabular}{|l|l|l|l|l|l|}
\hline \multirow{2}{*}{$\begin{array}{l}\text { Result } \\
\text { number }\end{array}$} & $\begin{array}{l}\text { Inter- } \\
\text { element } \\
\text { spacing }(d)\end{array}$ & \multicolumn{2}{|l}{$\begin{array}{l}\text { Beam width (in } \\
\text { degrees) in the } \\
\text { main lobe }\end{array}$} & \multicolumn{2}{l}{$\begin{array}{l}\text { MER (dB) in the } \\
\text { side lobe }\end{array}$} \\
\cline { 3 - 6 } & $N=5$ & $N=10$ & $N=5$ & $N=10$ \\
\hline 1 & $d=0.2 \lambda$ & $92^{\circ}$ & $51^{\circ}$ & -19.17 & -9.12 \\
\hline 2 & $d=0.3 \lambda$ & $54^{\circ}$ & $31^{\circ}$ & -6.93 & -11.65 \\
\hline 3 & $d=0.4 \lambda$ & $50^{\circ}$ & $29^{\circ}$ & -9.62 & -13.97 \\
\hline 4 & $d=0.5 \lambda$ & $48^{\circ}$ & $27^{\circ}$ & -12.1 & -11.9 \\
\hline 5 & $d=0.6 \lambda$ & $76^{\circ}$ & $54^{\circ}$ & -0.24 & -0.45 \\
\hline 6 & $d=0.7 \lambda$ & $75^{\circ}$ & $49^{\circ}$ & -0.11 & -0.75 \\
\hline
\end{tabular}

Research article

CIndian Society for Education and Environment (iSee)

"Wireless cellular network" http://www.indjst.org
Vol. 5 No. 4 (Apr 2012)

ISSN: 0974- 6846 in this method the interference is higher than that in CLMS and CGBF algorithms and SB technique. On the other hand, it can be seen that with $N=20$ the beam-forming method has better performance in comparison with the case $N=15$. In addition, we can see that the SINR of mobile user for $d=0.5 \lambda$ is better than that for $d=0.75 \lambda$. Also, it can be seen that the SINR of mobile user for the PPC is higher than that for the case of without using power control algorithm.

Fig. 10 shows the influence of path-loss parameter $\left(L_{p}\right)$ in USPS on the SINR of mobile user for SB, CGBF, and CLMS methods without using PC algorithm for $N=20, \mu=0.005$. The figure shows, as expected, a decrease in the path-loss parameter entails an increase in the interference and desired signal levels and, therefore, an improvement in system performance using antenna arrays in the base station. For example, at a SINR of $10 \mathrm{~dB}$ and for the CLMS algorithm, the number of interference users is, respectively, 13 and 18 users for $L_{p}=0.01 \mathrm{~dB} / \mathrm{m}$ and $L_{p}=0.005 \mathrm{~dB} / \mathrm{m}$.

Finally, in Fig.11, the mean-squared error for tracking of mobile user using two algorithms, recursive least squares (RLS) and CLMS, for parameter setting of $\mu=0.001,0.005, N=20, d=0.5 \lambda$ and Iteration=400 has been shown. It is worth saying that the weighting factor is equal to unity for the RLS method. Regarding the obtained curves, the RLS outperforms the CLMS due to having smaller steady state error. This is because CLMS utilizes a transient input and has Mis-Adjustment error. Moreover it is obvious for the CLMS that the bigger the step length $(\mu)$, the bigger the Mis-Adjustment error will be, and at the same time the smaller the convergence time we will have. Note that the convergence time for the RLS and CLMS, where $\mu=0.005$, are equal to 40 , twice than the number of elements of array antenna (Haykin, 1996). Also, the RLS method has more complexity in its structure than the CLMS method does.

\section{Conclusions}

In this paper, we studied adaptive beam-forming algorithms for tracking a mobile user in an urban environment in reverse link. The performance of the system is observed for uniformly spaced linear array by varying the number of elements in an array antenna and varying the inter-element spacing between the array elements without using power control and perfect power control. Based on the experimental results, we observed that in spite of existing interference signals from undesired users, the performance of adaptive beam-forming algorithms for perfect power control; especially, when there is no spatial aliasing $(0.4 \lambda \leq d \leq 0.5 \lambda)$ are 

number of interference users (M-1) for SB, CGBF, CLMS, ES methods

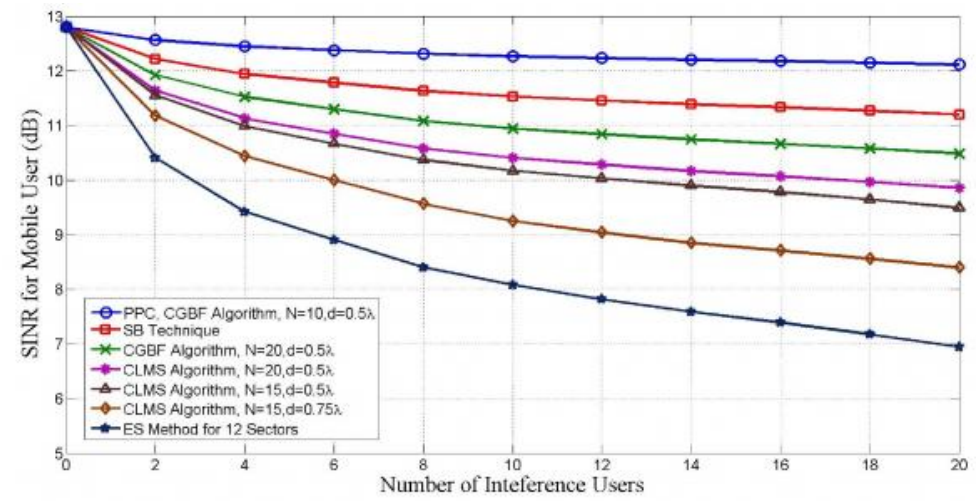

Fig. 10. Influence of path-loss parameter on the SINR of mobile user for SB, CGBF, CLMS, ES methods without using PC algorithm

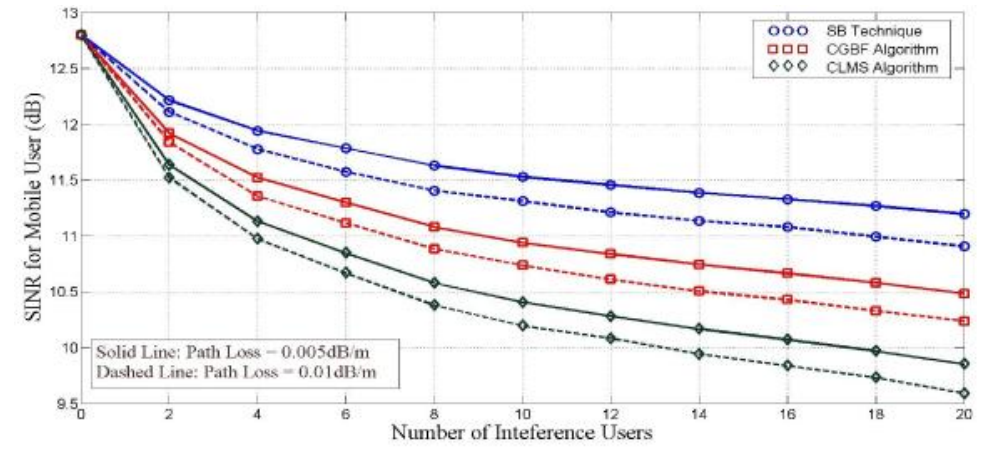

Fig.11. The mean-squared error for RLS and CLMS methods for $N=20$ and $d=\lambda / 2$

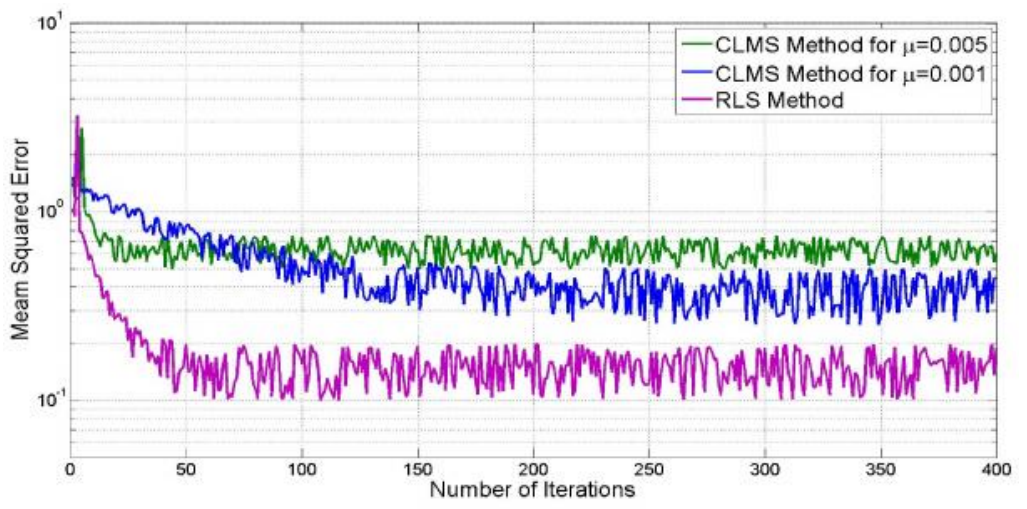

Fig.9. The SINR of mobile user in reverse link versus the

Vol. 5 No. 4 (Apr 2012) ISSN: 0974-6846

$\left(L_{p}\right)$ improves the SINR of mobile user.

References

1. Liberti JC and Rappaport TS (1999) Smart antennas for wireless communications. IS -95 and Third Generation CDMA Applications. New Jersey: Prentice Hall.

2. Shakir MZ and Durrani TS (2007) Narrow band beamforming algorithm for smart antennas. Int. Bhurban Conf. Appl. Sci \& Technol. pp. 49-54.

3. Riba J, Goldberg J and Vazquez G (1997) Robust beamforming for interference rejection in mobile communication. IEEE Transact. Signal Processing. 45, 271-275.

4. Dosaranian-Moghadam M, Bakhshi $H$, Dadashzadeh G and Rahmati P (2009) Adaptive beamforming method based on constrained LMS algorithm for tracking Mobile User. In: Proc. 2009 IEEE Global Mobile Congress, Shanghai, China. pp: 1-6.

5. Dosaranian-Moghadam M, Bakhshi $\mathrm{H}$ and Dadashzadeh G (2010) Joint centralized power control and cell sectoring for interference management in CDMA cellular systems in 2D Urban Environment. J. Wireless Sensor Network. 2(8), 599-605.

6. Dosaranian-Moghadam M, Bakhshi $\mathrm{H}$ and Dadashzadeh G (2010) Joint closed-loop power control and adaptive beamforming for wireless networks with antenna arrays in a 2D urban environment. J. Wireless Sensor Network. 2 (11), 869-878.

7. Gotsis KA, Siakavara K and Sahalos JN (2009) On the Direction of Arrival (DoA) Estimation for a switched-beam antenna system using neural networks. IEEE Transact. on Antennas \& Propagation. 57,1399-1411.

8. Rashid-Farrokhi F, Tassiulas L and Ray-Liu KJ Joint (1998) Optimal power control and beamforming in wireless networks using antenna arrays. IEEE Transact. on Commun. 46(10), 1313-1324.

9. Shakir MZ and Durrani TS (2007) Narrowband beamforming algorithm for smart antennas. Int. Bhurban Conf. Appl. Sci \& Technol. pp. 49-54.

10.Xinyu S, Xiaohua L and Jianjiang Z (2008) Robust adaptive beamforming based on maximum likelihood estimation. Int. Conf. Microwave \& Millimeter Wave Technol. 3, 1137-1140.

better than that for case of without using power control. On the other hand, it has been observed that the SINR of mobile user with the CGBF algorithm is higher than that with the CLMS algorithm, because in the CGBF algorithm, the IPI is removed whereas in CLMS algorithm it is not canceled. It has also been shown that the SINR of mobile user in the SB technique is lower than that in the CGBF algorithm. Also, our simulations show that when the number of the components of the array antenna $(N)$ increases, the SINR of mobile user increases. It has also been observed that decreasing the path loss parameter Research article (CIndian Society for Education and Environment (iSee)

11. Litva J and Kwok-Yeung T (1996) Digital beamforming in wireless communications. Artech House.

12. Haykin S (1996) Adaptive Filter Theory. $3^{\text {th }}$ ed., New Jersey: Prentice Hall.

13.Peterson RL, Ziemer RE and Borth DE (1995) SpreadSpectrum communications. Prentice-Hall.

14.Dosaranian-Moghadam M, Bakhshi $H$ and Dadashzadeh G (2010) Interference management for DS-CDMA systems through closed-loop power control,
M.D-Moghadam \& H.Bhakshi Indian J.Sci.Technol. 
base station assignment, and beamforming. $J$. Wireless Sensor Network. 2(6), 472-482.

15.Dosaranian-Moghadam M, Bakhshi $H$ and Dadashzadeh G (2010) Joint constrained LMS algorithm and base station assignment for DS-CDMA receiver in multipath fading channels. In: Proc. 2010 IEEE Wireless Commun., Networking \& Mobile Computing, Chengdu, China, Sep. 23-25. pp: 1-7.

16.Dosaranian-Moghadam M, Bakhshi H, Dadashzadeh G, and Godarzvand-Chegini M (2010) Joint closedloop power control and constrained LMS algorithm for DS-CDMA receiver in multipath fading channels. In: Proc. 2010 IEEE Global Mobile Congress, Shanghai, China, Oct. 18-19. pp: 1-8.

17.Dosaranian-Moghadam $M$, Bakhshi $H$ and Dadashzadeh G (2011) DS-CDMA cellular systems performance with base station assignment, power control error and beamforming over multipath fading. Int. J. Computer Networks \& Commun. 3(1), 185-202.

18. Rashid-Farrokhi F, Ray-Liu KJ and Tassiulas L (1998) Transmit beamforming and power control for cellular systems. IEEE J. Selected Areas in Commun. 16(8), 1437-1450.

19. Mohamed NA and Dunham JG (2002) A lowcomplexity combined antenna array and interference cancellation DS-CDMA receiver in multipath fading channels. IEEE J. Selected Areas in Commun. 20(2), 248-256.

20. Mohamed NA and Dunham JG (1999) Adaptive Beamforming for DS-CDMA using Conjugate Gradient Algorithm in a Multipath Fading Channel. In Proc. 1999 IEEE Emerging Technologies Symp., Dallas, TX, Apr. 12-13. pp: 859-63.

21.Dosaranian-Moghadam M, Bakhshi $H$, Dadashzadeh G and Godarzvand-Chegini M (2010) Joint Base Station Assignment, Power Control Error, and Adaptive Beamforming for DS-CDMA Cellular Systems in Multipath Fading Channels, In Proc. 2010 IEEE Global Mobile Congress, Shanghai, China, Oct. 18-19. pp: 1-7.

22.Dosaranian-Moghadam $M$, Bakhshi $H$ and Dadashzadeh G (2010) Interference management for DS-CDMA receiver through base station assignment in multipath fading channels. In: Proc. 2010 IEEE Int. Conf. on Wireless Commun., Networking \& Information Security, Beijing, China. pp: 257-263.

23. Corazza GE, De Maio G and Vatalaro F (1998) CDMA cellular systems performance with fading, shadowing, and imperfect power control. IEEE Transact. On Vehicular Technol. 47(2), 450-459.

Vehicular Technol. 47(2), 450-459. 